\title{
Is autoimmunity coming to a Fas(t) end?
}

\author{
Using bisindolylmaleimide to enhance the suicide program of activated lymphocytes is a novel therapeutic \\ approach to the treatment of autoimmune disease (pages 42-48 and 90-96).
}

C ONSIDERABLE EFFORTS ARE underway to improve our understanding of autoimmune disorders and to identify the underlying failure in immune system regulation. The goal of these efforts is to develop specific treatments that downregulate deleterious autoimmune reactions without affecting normal immune surveillance. Most strategies focus on either re-establishing immune tolerance to autoantigens or suppressing $\mathrm{T}$ Iymphocyte activity using immunosuppressive drugs, such as cyclosporin A, FK506 or rapamycin ${ }^{1}$. Such approaches, however, have their disadvantages. Tolerance induction requires the characterization of autoantigens involved at each stage of the autoimmune process. Immunosuppressive drug treatment is limited by the lack of antigen specificity, which results in general suppression of $\mathrm{T}$ cell immune function. Furthermore, prolonged immunosuppressive therapy is often accompanied by adverse side effects, such as nephrotoxicity ${ }^{1}$.

On page 42 of this issue, Zhou and colleagues ${ }^{2}$ suggest a novel strategy for the treatment of autoimmunity. This strategy is based on the observation that the total number of autoreactive $\mathrm{T}$ lymphocytes depends on a balance between the induction and proliferation of autoimmune $T$ cells and their elimination by activation of an internal suicide program. This program-termed activation induced cell death (AICD)-is initiated in activated lymphocytes following re-stimulation by antigen and involves expression of deathinducing ligands and receptors. Zhou et al. now report that the protein kinase $C$ (PKC) inhibitor bisindolylmaleimide VIII (Bis VIII) markedly enhances the sensitivity of T cells to AICD.

Specific interactions of the 'death' re ceptor and its ligand induce apoptosis in activated T cells, and the cellular 'corpses' are subsequently removed by phagocytic cells. In $\mathrm{CD}^{+} \mathrm{T}$ lymphocytes, AICD is preferentially mediated by the interaction of the Fas (CD95/Apo-1) receptor with its ligand (FasL/CD95L). By contrast, in CD8 ${ }^{+}$ $\mathrm{T}$ cells the TNF receptor/TNF $\alpha$ combination appears to be dominant ${ }^{3}$. When first activated, $T$ cells are resistant to AICD but they become sensitized during the effector phase of the immune response. Bis

\section{THOMAS BRUNNER \& \\ CHRISTOPH MUELLER}

VIII may promotecell death in T cells (and in non-lymphoid tumor cells) by interfering with inhibitors of apoptosis that block the Fas and TNF receptor-mediated cell death pathways.

In the Zhou study, treatment of rodents with Bis VIII in vivo abrogated autoantigen-specific immune responses as well as autoimmunity in two different models of $\mathrm{T}$ cell-mediated autoimmune disease-experimental allergic encephalitis (a model of multiple sclerosis) and adjuvant arthritis. This indicates that accel erated induction of the suicide program in T cells can limit (auto)antigendriven immune responses and that modulating apoptosis is an important way of regulating the immune system.

What is the molecular target of Bis VIII? Bisindolylmaleimides are potent inhibitors of PKC, a family of molecules that is known to mediate anti-apoptotic effects in many cell types ${ }^{4,5}$. This suggests that inactivation of PKC by Bis VIII is responsible for the sensitization of $T$ cells to death re- ceptor-induced apoptosis. However, many (but not all) PKC inhibitors exert such an effect, suggesting that either Bis VIII has additional targets or that only certain PKC isoforms are involved in blocking apoptosis. It is tempting to speculate that these PKC isoforms (which have yet to be identified) may promote resistance to 'death' receptor-induced apoptosis by activating signaling pathways that induce expression of anti-apoptotic genes (refs. $6,7)$. Alternatively, these PKC isoforms may promote phosphorylation of pre-existing anti-apoptotic molecules (such as $\mathrm{BCl}-2$ ) thus increasing the resistance of cells to apoptosis ${ }^{8}$.

Zhou and co-workers have demonstrated the beneficial effect of Bis VIII for the prevention or attenuation of autoimmune disease in two experimental $\mathrm{CD}^{+} \mathrm{T}$ cell-mediated autoimmune disorders. However, their data indicate that immunopathological reactions that involve cytotoxic $\mathrm{CD}^{+} \mathrm{T}$ cells may also be attenuated by BisVIII because this drug promotes TNF $\alpha$-mediated apoptosis (an essential component of AICD in CD8 ${ }^{+} \mathrm{T}$ cells).

The Zhou study demonstrates that the simplenotion of enhancing immune regu-

\section{$\begin{array}{llc}a & b & c\end{array}$}

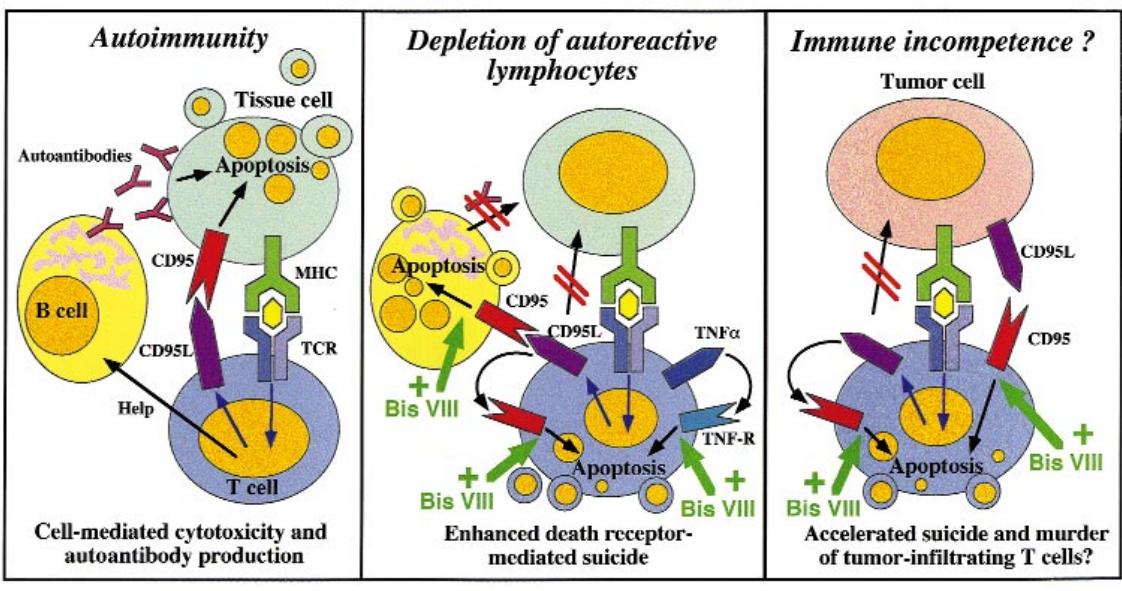

a, Autoreactive T cells are activated by self antigen present on tissue cells. Stimulated T cells produce cytokines, express costimulatory ligands and provide B cell help to support the production of autoantibodies. In addition, T cells develop cytotoxic effector functions and express Fas ligand (FasL/CD95L), which induces apoptosis of target cells. b, Upon recognition of self antigens, autoreactive T cells express 'death' receptors (Fas/CD95; TNFR) and their ligands (FasL/CD95L; TNF $\alpha$ ). Treatment with Bis VIII sensitizes autoreactive T Iymphocytes to apoptosis via the 'death' receptor pathway. c, Tumor cells present antigen to tumor infiltrating T cells that express the 'death' receptor and its ligand. Upon sensitization with Bis VIII, activated T cells undergo accelerated apoptotic cell death. The demise of tumor infiltrating $T$ cells is further enhanced by tumor cells bearing FasL (CD95L), which are resistant to Bis VIII because they do not express the Fas (CD95) receptor. 


\section{Cytotoxic T cells: Double-barreled shot guns}

The killing of target cells by cytotoxic T lymphocytes is an efficient way for the immune system to eliminate virus-infected cells, intracellular parasites and tumor cells. T cells and Natural Killer (NK) cells have two major effector mechanisms: (1) the expression of FasL (CD95L), which induces cells expressing the Fas receptor to undergo apoptosis and (2) re lease of perforin and granzymes from cytotoxic granules. These two effector systems have in common the fact that they exploit the cell's own apoptosis machinery. Engagement of FasL with its receptor or the release of granzyme/ perforin results in the immediate activation of the caspase (protease) cascade ${ }^{12}$, a crucial step in the apoptosis pathway.

In this issue of Nature M edicine, Bossi and Griffiths ${ }^{13}$ now report that these cytotoxic effector mechanisms have even more in common. Activated cytotoxic T lymphocytes (both $\mathrm{CD}^{+}$and $\mathrm{CD}^{+}$) as well as NK cells can store preformed FasL in lytic granules that may also contain granzymes, perforin and other granule proteins. Thus, as with perforin and granzyme, FasL can be rapidly brought to the cell surface by degranulation following antigen stimulation. FasL then induces the antigen presenting target cell to undergo apoptosis. Similar findings have been reported for $\mathrm{TNF} \alpha$, which is stored in mast cells and is released after IgE receptor activation ${ }^{14}$. The storage of preformed 'death' ligands in the granules of primed cytotoxic $T$ cells enables a quicker response to antigen re-stimulation than would be possible with de novo protein synthesis. In this way, more targets can be killed more efficiently in less time.

This study goes on to show that FasL-mediated cytotoxicity is regulated at the post-transcriptional as well as the transcriptional level. It is known that the cytoplasmic tail of FasL interacts with distinct domains of Fyn tyrosine kinase, suggesting that the T cell receptormediated appearance of FasL at the cell surface may involve a tyrosine kinase signaling pathway ${ }^{15}$. Whereas activation leads to a transient increase in cell surface FasL, its subsequent rapid downregulation may be an important mechanism to prevent bystander killing of innocent Fas receptor-positive targets. It remains to be determined whether preformed FasL and newly synthesized FasL are preferentially involved in distinct cellular functions, such as paracrine killing of Fas-positive targets and autocrine activation-induced suicide of T cells, respectively ${ }^{16}$.

T.B. \& C.M .

latory mechanisms, which already exist but are impaired in autoimmune disease, is effective. This approach does not require the identification of autoantigens because only activated T cells appear to be affected by Bis VIII treatment. Because undesirable $T$ cell-mediated immune responses are the underlying cause of many other pathologies, one can envision that a similar strategy may prevent organ transplant rejection or graft-versus-host disease.

Despite these promising findings, extensive research is still needed to define the limits of such a therapeutic approach. In clinical settings, autoimmune disease generally becomes evident with tissue destruction, which occurs long after the initial induction of autoimmune effector cells. Hence, this situation is fundamentally different from that in experimental systems where autoimmunity is treated at the onset of the initial autoimmune reaction, before there is clinical evidence of disease.

Bis VIII affects the survival of activated cells irrespective of their antigen-specificity and without disturbing the initial activation of naive $T$ cells. Therefore, in contrast to currently available immunosuppressive drugs, Bis VIII should not paralyze the immune system but rather should target all autoantigen-specific and activated lymphocytes that emerge during the establishing of an autoimmune disorder. Mounting an immune response against infectious agents should still be possible, although the response may be limited because Bis VIII will decrease the half life of activated lymphocytes.

Despite the bright outlook for Bis VIII therapy, some words of caution are in order. Increasing the Fas-mediated apoptosis of autoreactive $T$ cells and tumor cells that express Fas receptor is certainly a worthy goal but are there situations where accelerated induction of apoptosis may be deleterious? Although impairment of humoral and cellular immune responses may be minimal compared to the dramatic effects of immunosuppressive drugs, Bis VIII may induce certain cell types to undergo 'premature' apoptosis. Hepatocytes and intestinal epithelial cells are known to be highly susceptible to apoptosis following Fas or TNF receptor activation $^{9,10}$. Prolonged Bis VIII therapy may increase apoptotic cell loss in the liver and the intestine resulting in severe side effects. Furthermore, in light of recent observations that tumor cells fight off attacking $T$ cells by inducing them to undergo Fas-mediated apoptosis, tumor infiltrating $\mathrm{T}$ cells may end up murdered killers following Bis VIII treatment ${ }^{11}$. Thus, apoptosis remains a double-edged sword. Nevertheless, even if the path to successful treatment of autoimmunity is full of pitfalls, without new approaches there will be no cure.

Acknowledgement

We thank Andreas Kappeler for helpful comments.

1. Chatenoud, L. Tolerogenic antibodies and fusion proteins to prevent graft rejection and treat autoimmunity. Mol. Med. Today 4, 25-30 (1998)

2. Zhou, T. et al. Bisindolylmaleimide VIII facilitates Fas mediated apoptosis and inhibits T-cell mediated autoimmune disease. Nature M ed. 5, 42-48 (1999).

3. Lenardo, M.J. Fas and the art of lymphocyte maintenance. J.Exp.M ed. 183, 721-724 (1996).

4. Su, X. et al. Defective expression of hematopoietic cell protein tyrosine phosphatase (HCP) in lymphoid cells blocks Fas-mediated apoptosis. Immunity 2, 353-362 (1995).

5. Ruiz-Ruiz, M.C., Izquirdo, M., de Murcia, G. \& LopezRivas, A. Activation of protein kinase C attenuates early signals in Fas-mediated apoptosis. Eur. J. Immunol. 27, 1442-1450 (1997).

6. Van Antwerp, D.J., Martin, S.J., Kafri, T., Green, D.R. \& Verma, I.M. Suppression of TNF-alpha-induced apoptosis by NF-kappaB. Science 274, 787-789 (1996).

7. Wang, C.Y., Mayo, M.W., Komeluk, R.G., Goeddel, D.V. \& Baldwin, A.S.J. NF-kappaB anti-apoptosis: induction of TRAF1 and TRAF2 and c-IAP1 and C-IAP2 to suppress caspase-8 activation. Science $\mathbf{2 8 1}$ 1680-1683 (1998).

8. Ruvolo, P.P., Deng, X., Carr, B.K. \& May, W.S. A functional role for mitochondrial protein kinase $C$ alpha in Bcl-2 phosphorylation and suppression of apoptosis. J. Biol. Chem. 273, 25436-25442 (1998).

9. Ogasawara, J. et al. Lethal effect of the anti-Fas antibody in mice. Nature 364, 806-809 (1993).

10. Guy-Grand, D., DiSanto, J.P., Henchoz, P., MalassisSeris, M. \& Vassalli, P. Small bowel enteropathy: role of intraepithelial lymphocytes and of cytokines (IL-12, IFN-gamma, TNF) in the induction of epithelial cell death and renewal. Eur. J. Immunol. 28, 730-744 (1998).

11. Walker, P.R, Saas, P. \& Dietrich, P.Y. Role of Fas ligand (CD95L) in immune escape: the tumor cell strikes back. J. Immunol. 158, 4521-4524 (1997).

12. Froelich, C.J., Dixit, V.M. \& Yang, X. Lymphocyte granule-mediated apoptosis: matters of viral mimicry and deadly proteases. Immunol. Today 19, 30-36 (1998).

13. Bossi, G. \& Griffiths, G.M. Degranulation plays an essential part in regulating cell surface expression of Fas ligand in T cells and Natural Killer cells. Nature M ed. $\mathbf{5}$, 90-96 (1999)

14. Gordon, J.R. \& Galli, S.J. Release of both preformed and newly synthesized tumor necrosis factor alpha (TNF-alpha)/cachectin by mouse mast cells stimulated via the Fc epsilon R. A mechanism for the sustained action of mast cell-derived TNF-alpha during IgE-dependent biological responses. J. Exp. Med. 174, 103-107 (1991).

15. Hahne, M., Lowin, B., Peitsch, M., Becker, K. \& Tschopp, J. Interaction of peptides derived from Fas ligand with the Fyn-SH3 domain. FEBS Lett. 373, 265-268 (1995).

16. Su, X. et al. Autocrine and paracrine apoptosis are me diated by differential regulation of Fas ligand activity in two distinct Jurkat T cell populations. J. Immunol. 160, 5288-5293 (1998)

Institute of Pathology

University of Berne

CH-3010 Berne, Switzerland

email: brunnert@patho.unibe.ch 